Debbie Massey MSc, Ba (hons) RGN ENB 249 PGCE Senior Lecture

School of Health \& Social care

Oxford Brookes University

Marston Road

Oxford

OX3 OFL

Email dlmassey@brookes.ac.uk 



\section{Cardiac Assessment}

Over the last fifteen years numerous political drivers have paved the way for the development of new and innovative models of practice within cardiac care. Many of these new models of practice requires practitioners to perform detailed and comprehensive cardiac assessment.

This paper offers practitioners an opportunity to develop and augment their knowledge and understanding of cardiac auscultation It suggests a systematic model that may be used in clinical practice.

\section{Key words}

Cardiac assessment, clinical assessment, cardiac auscultation holistic care, advanced roles.

\section{Introduction}

Nursing within all specialties continues to face unprecedented growth and development. Since the early 1990s there has been a proliferation and expansion of nurses' roles. The impetus for these developments has been multifactoral but it would appear that within cardiac care there are three main drivers. Firstly the reduction in junior doctors' hours (NHSME 1991) has encouraged nurses to develop roles that have traditionally been performed by medical staff. The Scope of Professional Practice (UKCC1992) has been significant in enabling nurses' role expansion and development. The implementation of the National Service Frameworks paved the way for innovative new models of practice to be developed and fostered the creation of new roles for practitioners, for example the development of nurse led thrombolysis (Quinn 1995). Many of these new roles and developments required practitioners to develop history taking and assessment skills (Hind et al 1999). It is increasingly acknowledged that the development of history taking and assessment skills is a key component of role development. The ability to undertake and document a clear and concise systems assessment constitutes a major aspect of the clinical practitioner's role. By expanding and developing this skill practitioners can ensure patients receive timely and appropriate interventions. 
As practitioners enter the realm of patient assessment, so begins the integration of essential elements of clinical care, observation, empathic listening and the techniques of examining and assessing different body systems and the development of the process of clinical reasoning. Critical care practitioners are increasingly performing respiratory assessment in the critically ill patient (Cox and McGrath 1999).

However, a literature search using Medline and CINAHL databases revealed limited publications regarding cardiac assessment with only three published articles found: two American articles written in 1988 which concentrate specifically on auscultation (Becker \& Stevens 1988 and Miracle 1988) and one British article aimed at critical care practitioners (McGrath and Cox 1998). Given the expansion and developments of nurses' roles and responsibilities within all aspect of cardiac care it would appear timely and appropriate to revisit this topic and ensure its applicability to all practitioners caring for patients with a cardiovascular complaint.

The aim of this paper is to enable practitioners to develop a more holistic approach to the clinical assessment of the cardiac patient. It aims to develop practitioners' knowledge and understanding of cardiac assessment and its applicability to the clinical environment. It will suggest a systematic model to utilise during auscultation of the cardiac system. Part one of this paper introduced practitioners to the techniques of inspection and palpation (Massey 2006). This paper introduces a systematic model that may be utilised during cardiac auscultation.

A significance amount of information can be obtained through auscultation of the cardiac system and when performed correctly it can be a highly rewarding and valuable diagnostic tool. However Cardiac auscultation requires a logical and methodical approach, a thorough understanding of cardiac anatomy and physiology and the cardiac cycle.

Before auscultation is attempted it is imperative that a good quality stethoscope is available. Although the human ear is able to hear sounds up to $20.000 \mathrm{Hertz}(\mathrm{Hz})$ it is most sensitive to sounds to 1,000 to $5,000 \mathrm{HZ}$. The frequency of most heart sounds is less than $1,000 \mathrm{~Hz}$ (Woods 2005). Therefore the aim of the stethoscope is to transmit these sounds clearly to the ear. Stethoscopes consist of earpieces, tubing and the chest piece. The tubing should not be longer than $30 \mathrm{~cm}$. This ensures that the heart sounds are not diluted. There are two basic types of chest pieces: the diaphragm and the bell. The diaphragm enhances higher frequency sounds and 
filters out lower frequency sounds. The bell filters out high frequency sounds and augments low frequency sounds.

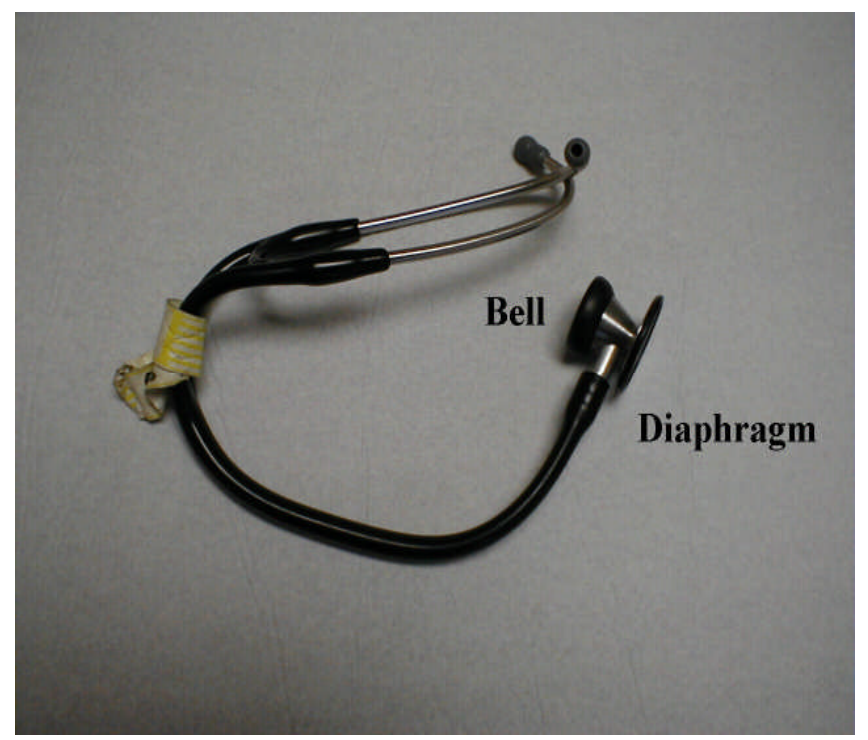

Before cardiac auscultation commences it is important that the patient is fully prepared. Ensure the room is as quiet as possible. Attempting to auscultate through clothing may distort heart sounds. The precordium therefore needs to be exposed during auscultation. Ensure that the patient remains warm throughout the procedure and that the stethoscope is warm. It is also important to fully explain the procedure to the patient.

\section{Auscultation of the Heart}

There are normally two heart sounds called the first and second heart sounds (Tortora \& Grabowski 2000). The first sound results from closure of the mitral and tricuspid valves. The second heart sound results from closure of the aortic and pulmonic valves (Bickely 2003). The first heart sound is denoted as $S 1$ and the second heart sound as S2. S1 coincides with the beginning of ventricular systole whilst S2 coincides with the end of ventricular systole (Bickely 2003). S1 is lower pitched than S2 and gives a lubb sound. S2 gives the characteristic dubb sound.

When auscultatating for heart sounds it is important that all areas of the precordium are auscultatated. Normal heart sounds indicate events in the cardiac cycle and these events are reflected in the different areas of the chest wall. Auscultation sites are identified by the names of heart valves. However these areas are not located 
directly over the valves, rather they are located along the pathway that blood flows through the heart chambers and valves. There are four different sites and these are the aortic area, pulmonic area tricuspid area and the mitral valve area (Bickley 2003) (see Fig 2). There is no consensus on what constitutes the correct auscultation route however what is important is that the procedure is systematic and methodical and that all four sites are listened to with the bell and the diaphragm. This paper suggests a methodical and systematic model that may be useful to utilise in the practice setting.

Fig 2

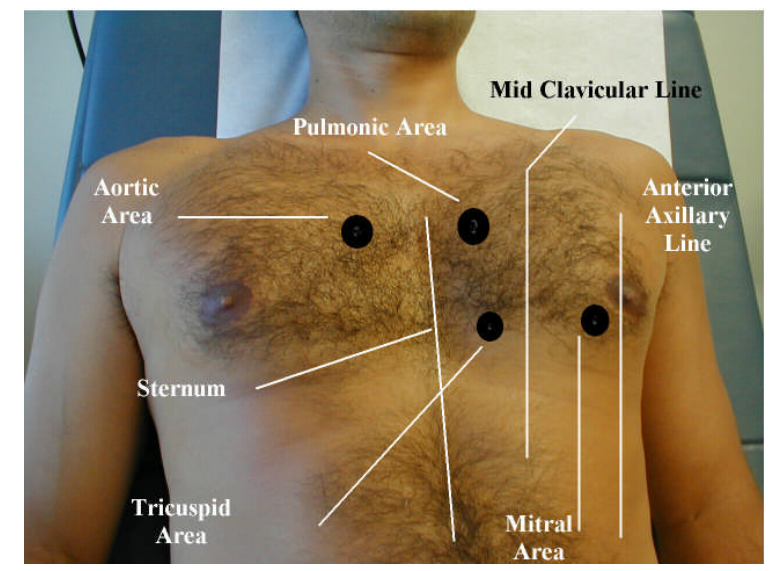

Firstly with the diaphragm of the stethoscope listen over the 2nd right intercostal space close to the sternum - the region of the aortic valve. Then move the stethoscope to the other side of the sternum and listen in the 2nd left intercostal space - the location of the pulmonic valve. Move the stethoscope down along the sternum and listen over the left 4th intercostal space: the region of the tricuspid valve. Finally, position the stethoscope over the 5th intercostal space, left midclavicular line and auscultate the mitral area at the apex of the heart. Repeat this sequence with the bell of the stethoscope

In each area, listen specifically for S1 and then S2. S1 will be loudest over the left 4th intercostal space (mitral/tricuspid valve areas). S2 is loudest over the aortic and pulmonic areas; the $2^{\text {nd }}$ right and left intercostal spaces (Woods 2005). The first heart sound, S1 is heard loudest at the apex of the heart. The intensity of S1 depends on the mobility of the mitral and tricuspid valve leaflets and the rate of ventricular upstroke (Woods 2005). A loud S1 occurs in mitral stenosis as the narrowed valve shuts suddenly producing a loud first heart sound. A soft $S 1$ is most frequently the result of poor conduction of sound through the chest wall but other causes include a 
fixed or immobile valve or a slow heart rate, which allows the atrioventricular valves to float back into position before the ventricular systole (Woods 2005). The intensity of $S 1$ will vary beat to beat in atrial fibrillation because diastolic filling time is not constant.

$\mathrm{S} 2$ is a shorter, higher pitched and louder than $\mathrm{S} 1$ and is loudest at the base at the heart over the pulmonic and aortic areas. The intensity of $\mathrm{S} 2$ is determined by pressures in the receiving vessels and the mobility of the aortic and pulmonic valve leaflets and the size of the aortic root (Woods 2005). S2 will be increased in pulmonary or systemic hypertension or ascending aortic aneurysm. S2 will be soft and intensity diminished in the presence of heart failure, myocardial infarction and stenosis of the pulmonic or aortic valve (Bickley 2003). This information should aid the practitioner to decide which sound is produced by closure of the mitral/tricuspid and which by the aortic/pulmonic valves and therefore when systole and diastole

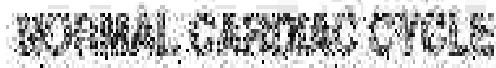

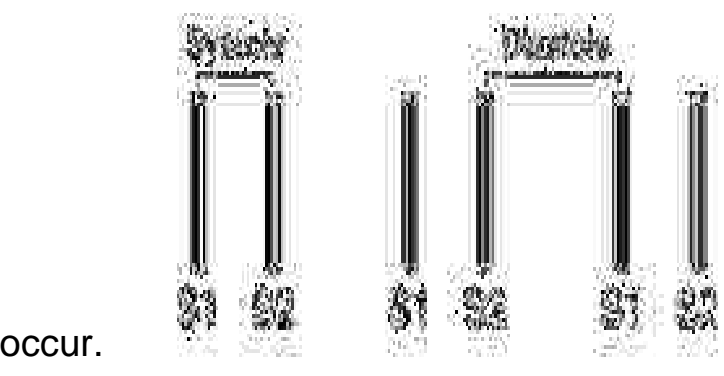

S2 may be split (Marsh 1999). This is a normal finding and is refered to as physiologic splitting. S2 is made up of 2 components; aortic (A2) and pulmonic (P2) valve closure. Inspiration decreases thoracic pressure and therefore increases peripheral vascular return and venous return to the heart is augmented and pulmonic valve closure is delayed (Cox and Roper 2005). The right side of the heart therefore takes longer to fill so pulmonic valve closure is delayed. Thus in inspiration it may be possible to hear a split S2. This is heard best in the pulmonic area the $2^{\text {nd }}$ left intercostal space. Asking the patient to take a deep breath and hold it gives more time to identify this phenomenon. The two components of S1 (mitral and tricuspid valve closure) occur so close together that splitting is not appreciated (Cox and Roper 2005). 


\section{Extra heart sounds}

Following auscultation of S1 \& S2 extra heart sounds should be listened for. Extra sounds may be present in children and adults. However in older adults over the age of 20 they represent pathology (Mangione 2000). These extra sounds are referred to as S3 \& S4. S3 is one of the first clinical sound associated with left ventricular failure and is caused by vibrations occurring during rapid passive ventricular filling as blood enters a non compliant left ventricle (Woods 2005). A physiological S3 may also be audible in high output states in which rapid ventricular expansion is caused by an increase in blood volume. Anaemia, fever, pregnancy and thyrotoxicosis are some of the conditions that may cause high output states. S3 is best heard at the apex the fifth intercostal space in the left lateral position (Bickely 2003). This position brings the left ventricle closer to the chest wall thus allowing improved transmission of S3 (fig 2). Because S3 is a low frequency sound it is best heard with the bell of the stethoscope.

S4 occurs after atrial contraction as blood is ejected into a stiff non-compliant left ventricle. Whereas S3 is indicative of systolic dysfunction S4 signifies diastolic dysfunction. S4 occurs immediately following diastole and is therefore heard immediately before S1. S4 is most frequently associated with left ventricular hypertrophy that is the result of long standing hypertension or following a myocardial infarction (Woods 2005). It is common in the elderly as a result of decreased left ventricular compliance. Because atrial contraction is necessary to produce an S4 this sound cannot be auscultated in the presence of atrial fibrillation. Like S3, S4 is a low frequency sound and is best heard with the bell of the stethoscope. Listen for S4 at the apex the fifth intercostal space in the left lateral position. If tachycardia is present then a summation gallop is heard - S3 and S4 fuse together in mid diastole to produce one loud diastolic sound. The summation gallop resembles the sound of a galloping horse. 


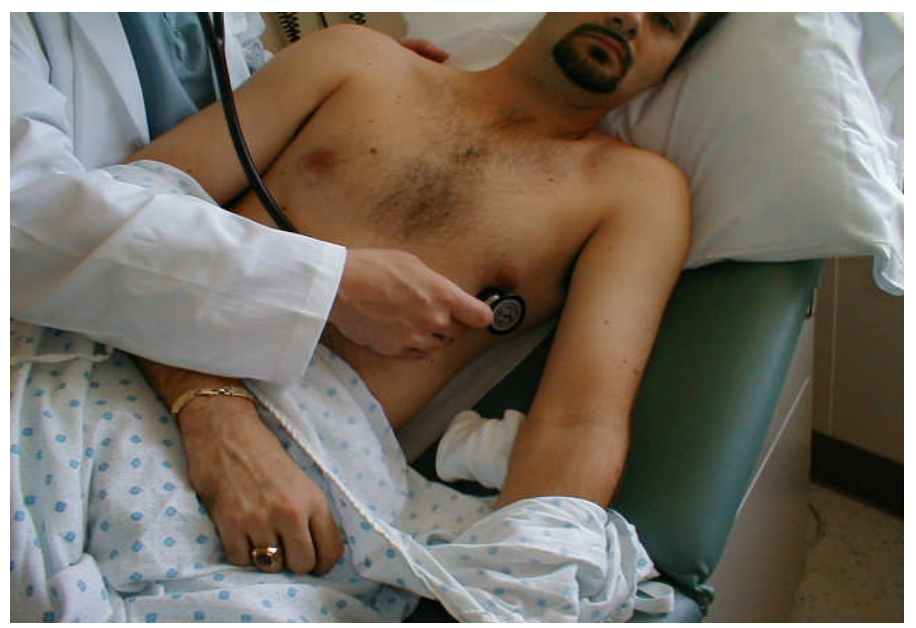

Fig 2 left lateral position

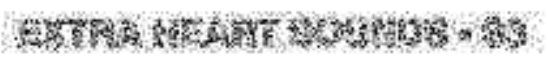
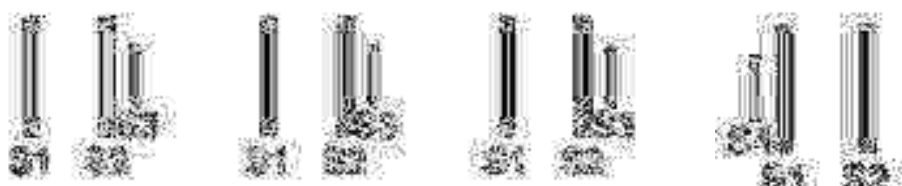

(2) 20:
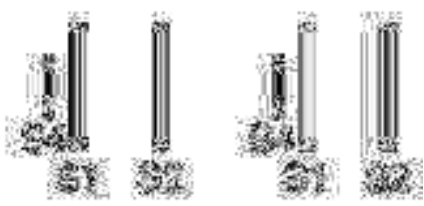

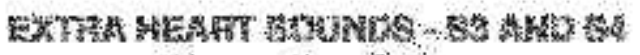

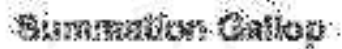

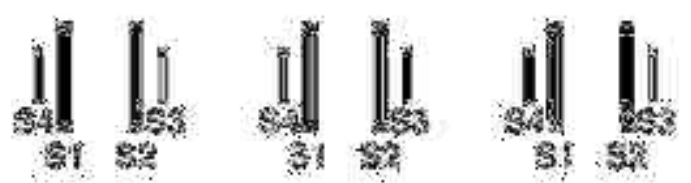

\section{Murmurs}

After auscultating $\mathrm{S} 1$ and $\mathrm{S} 2$ and assessing if any extra heart sounds are present ausculate for murmurs. A heart murmur is an abnormal sound heard during auscultation. Murmurs can be either physiological or benign. Murmurs are caused by turbulent blood flow. There are three main causes of murmurs (Becker \& Stevens 
1988). Firstly, high blood flow through a normal valve for example fever, pregnancy thyrotoxicosis and other conditions associated with high output states. Secondly, blood flow through a stenotic valve and thirdly backflow of blood through an incompetent or regurgitatant valve. Like all aspects of the cardiac examination the key to successful assessment and interpretation of a cardiac murmur is a methodical and systematic approach. It is imperative that murmurs are described carefully and accurately to enable easy recognition of changes in the murmur. Seven characteristics are used to describe murmurs (Woods 2005).

- Location $\sim$ this is the anatomic area on the precordium where the murmur is heard best

- Intensity $\sim$ this refers to the murmur's loudness. The Levine grading scale (Bickely 2003) should be used to describe and document a murmur's intensity.

- I/IV: Barely audible through the stethoscope, very faint

- II/VI: Audible as soon as the stethoscope is applied to the chest but is quiet and faint

- III/VI: The murmur is easily heard and is moderately loud

- IV/VI: A moderately loud murmur that may be accompanied by a palpable vibration called a thrill.

- V/VI: The murmur is audible even when only the edge of the stethoscope touches the chest and is almost always accompanied by a thrill

- VI/VI: A murmur so loud that it is heard with the naked ear. It is always accompanied by a thrill and often radiates to distant structures.

- Duration is the length of time the murmur is heard through systole or diastole. It can be described as long or short

- Pitch or frequency $\sim$ this can be high or low.

- Quality the murmur's quality is determined by the frequencies that produce the sound. Thus the murmur may be described as harsh, blowing, rumbling, or scratchy (Mangione 2001).

- Timing timing refers to when the murmur occurs in the cardiac cycle. Thus the murmur can be described as systolic or diastolic. Murmurs can be further classified according to their timing within the phase of the cardiac cycle. For 
example a murmur may be described as holosystolic meaning that it is present throughout the whole of systole or late diastolic or midsystolic.

- Configuration this final characteristic refers to the shape or pattern of the murmur as it is recorded on a phonocardiogram. A murmur's configuration is defined by changes in intensity during systole or diastole and is determined by the blood flow across pressure gradients. This characteristic is often represented and written as a waveform. A crescendo murmur gradually increases in intensity as the pressure gradient increases. A decrescendo murmur gradually decreases in intensity as the pressure gradient increases. A crescendo-decrescendo murmur also called a diamond shaped murmur initially increases in intensity and then decreases. Finally, a plateau shaped murmur remains in equal intensity.

During cardiac assessment it is important to differentiate between benign and pathological murmurs. Mangione (2001) suggests that applying a number of rules may help with this differentiation. Firstly judge a murmur by the company it keeps. Thus a murmur that keeps bad company such as an abnormal pulse, an abnormal chest $\mathrm{x}$-ray and abnormal electrocardiogram or any other cardiac symptoms should be considered pathological unless proven otherwise. Secondly a diminished or absent S2 is indicative of a poorly moving and abnormal semilunar valve and this finding is indicative of a pathology. Thirdly all diastolic murmurs are pathological, all holosytolic, late systolic murmurs and all continuous murmurs are pathological.

The four most common types of murmurs heard on auscultation and encountered in clinical practice are the murmurs of aortic stenosis, aortic regurgitation, mitral stenosis and mitral regurgitation. These will now be discussed and related to the auscultatory findings.

\section{Identifying the most common cardiac murmurs: Systolic Murmurs}

Aortic Stenosis is an ejection systolic murmur. Aortic stenosis can be congenital, or acquired as a result of degenerative or rheumatic heart disease (Woods 2005). Aortic stenosis is best heard over the base of the heart in the aortic area over the second right intercostal space. The murmur of aortic stenosis is medium pitch and can thus be heard equally well with the bell or the diaphragm of the stethoscope. The 
configuration of the murmur of aortic stenosis is crescendo/decrescendo configuration and is described as harsh or rasping (Mangione 2001). The murmur may also vary in intensity from a soft grade II/IV to a rough IV/VI. The murmur of aortic stensois may radiate towards the right side of the neck, the right shoulder. There may also be a palpable thrill over the aortic area.

Mitral regurgitation, also termed mitral insufficiency can be either chronic or acute. Most common causes of acute mitral regurgitation include chordal rupture, trauma, myocardial infarction or infective endocarditis (Woods 2005). Chronic mitral regurgitation is often the result of rheumatic heart disease (Marsh 1999). On auscultation the most easily noted characteristic of either acute or chronic mitral regurgitation is an holosystolic murmur heard best at the mitral area the 5th intercostal space, left midclavicular line. The murmur of mitral regurgitation often radiates to the axilla. The configuration, intensity and pitch of mitral regurgitation varies depending of the nature of the underlying pathology

\section{Diastolic Murmurs}

Aortic Regurgitation may be the result of Rheumatic fever or infective endocarditis which may cause destruction of the valve leaflets. Aortic regurgitation may also be the result of a congenital malformed valve. The typical murmur of aortic regurgitation is an early diastolic decrescendo murmur best heard in the third or fourth intercostal space over the aortic area (Marsh 1999). The murmur of aortic regurgitation is described as a high-pitched blowing murmur heard best with the diaphragm of the stethoscope and it has a decrescendo configuration (Bickely 2003).

\section{Mitral Stenosis}

The predominate cause of mitral stenosis is rheumatic valve disease (Bickely 2003). The murmur of mitral stenosis is best heard with the bell of the stethoscope at the apex of the heart over the mitral area, the fifth left intercostal space (Bickley 2003). The murmur of mitral stenosis is low pitched and has a rumbling quality. In severe mitral stenosis on auscultation there are four classic findings (Woods 2005). Firstly, an accentuated S1. Secondly an opening diastolic snap, thirdly, a mid diastolic rumble heard best at the apex and fourthly, an increased pulmonic S2 associated with pulmonary hypertension. The murmur of mitral stenosis has a crescendodecrescendo configuration. 


\section{Conclusion}

Within cardiac nursing, political drivers and advances in technology are compelling nurses to develop and expand their scope of practice. Increasingly, developing complex assessment skills is seen as an imperative for practitioners located within the cardiac care environment. This paper has presented a systematic model to apply to cardiac auscultation that practitioners may find useful to adopt within their clinical practice. A thorough and detailed cardiac assessment of patients is essential to ensuring a holistic and comprehensive model of care is delivered.

\section{Key Points}

- Nurses are increasingly developing complex and advanced roles

- Respiratory assessment is now considered integral to the scope of practice of the critical care practitioner

- There is a paucity of literature relating to cardiac assessment.

- Practitioners should begin to incorporate cardiac assessment into their repertoire of clinical skills

\section{References}

Cox N,T \& Roper T,A (2005) Clinical Skills Oxford University Press. Oxford England 
Department of Health NHS Management Executive (1991) Junior Doctors the New Deal. London

Becker K, L \& Steven S, A (1988) Get in Tune with Cardiac Assessment. Part 1. Nursing 3 52-55

Bickley L, S (2003) Bates Guide to Physical Examination and History Taking (8 ${ }^{\mathrm{TH}}$ edition) Lippincott Williams \& Wilkin Philadelphia

Cox C, L \& McGrath A (1998) Respiratory Assessment in Critical Care Units. Intensive and Critical Care Nursing 15, 226-234.

Hind M, Jackson D, Anderson, C Fulbrook, P Galvin K, Frost S (1999) Exploring the expanded role of the nurses in critical care. Intensive and Critical Care Nursing 15, 147-153.

Mangione S (2001) Physical Diagnosis Secrets Hanley \& Belfus Philadelphia

Marsh J (1999) History and Examination Mosby's Crash Course. Mosby International London

McGrath A \& Cox C, L (1998) Cardiac and Circulatory Assessment in Intensive Care Units, Intensive and Critical Care Nursing 14, 226-234.

Miracle V, A (1988) Get in Tune with Cardiac Assessment. Part 2. Nursing 4 42-47

Place G, \& Graham S (2000) Non Invasive Vital Organ Assessment. Nursing Times Plus 96 6-9

Quinn T (1995) Can nurses safely assess suitability for thrombolytic therapy? A pilot study Intensive and Critical Care Nursing 11 (3) 156-129

Seidel H,M, Ball J,W Dains J,E \& Benedict G,W (1995) Mosby's Physical Examination Handbook Mosby St Louis Missouri

Tortora G, T \& Grabowski S, R (2000) Principles of Anatomy and Physiology (9 ${ }^{\text {th }}$ edition) John Wiley \& Sons New York 
United Kingdom Central Council of Nursing and Health Visiting (1992) The Scope of Professional Practice. UKKCC London

Woods S, L Frorlicher E, S, S \& Adams S (2005) Cardiac Nursing (5 ${ }^{\text {th }}$ ed) Lippincott Philadelphia 